Recepción: 15 / 04 / 2017

Aceptación: 01 / 05 / 2017

Ciencias Médicas

Publicación: 15 / 05 / 2017

Reporte de Caso

\title{
Cáncer de colon y recto: Reporte de caso
}

\author{
Cancer of the colon and rectum: Case report
}

\section{Cancro do cólon e do recto: Relato de caso}

Franklin E. Encalada-Calero

f.encalada@hotmail.com

Nelson X. Ramirez-García " $\underline{\text { xavierramirez1958@gmail.com }}$

Marlon K. López-Chinga "' marlonlopchinga@gmail.com

Correspondencia: f.encalada@hotmail.com

Magister en Diseño Curricular; Especialista en Cirugía General; Medico; Doctor en Medicina y Cirugía; Cirujano General, Docente de la Universidad de Guayaquil, Médico Tratante del Hospital Teodoro Maldonado Carbo, Ecuador.

II. Especialista en Cirugía General; Doctor en Medicina y Cirugía; Cirujano General, Docente de la Universidad de Guayaquil, Médico Tratante del Hospital Teodoro Maldonado Carbo, Ecuador.

III. Médico; Médico Residente Hospital Teodoro Maldonado, Ecuador. 


\title{
Resumen
}

El cáncer de colon y recto es la segunda causa de muerte en países industrializados y en nuestro país es la quinta en incidencia de patologías malignas. El estudio constante de las bases genéticas y moleculares nos abre nuevas esperanzas hacia el tratamiento o a su vez la explicación de por qué fallo un esquema terapéutico. La piedra angular del tratamiento es la cirugía sin embargo en los últimos meses se ha cuestionado debido a que se da quimio-radioterapia (ver y esperar) ya que el 50 $\%$ de los pacientes tienen una respuesta adecuada.

Palabras clave: Colon; cáncer; recto.

\begin{abstract}
Colon and rectal cancer is the second leading cause of death in industrialized countries, and our country is the fifth leading cause of malignancies. The constant study of the genetic and molecular bases opens our hopes for the treatment or, in turn, the explanation of why a therapeutic scheme failed. The cornerstone of the treatment is surgery however in the last months it has been questioned because of chemoradiotherapy (seeing and waiting) since $50 \%$ of patients have an adequate response.
\end{abstract}

Key words: Colon; cancer; rectum.

\section{Resumo}

Cólon e câncer retal é a segunda principal causa de morte nos países industrializados, e nosso país é a quinta principal causa de malignidades. O constante estudo das bases genéticas e moleculares abre nossas esperanças para o tratamento ou, por sua vez, a explicação de por que um esquema terapêutico falhou. A pedra angular do tratamento é a cirurgia no entanto nos últimos meses tem sido questionada por quimiorradioterapia (ver e esperar) uma vez que 50\% dos pacientes têm uma resposta adequada.

Palavras chave: Cólon; câncer; reto. 


\section{Introducción.}

El cáncer colorrectal (CCR), es una entidad frecuente y letal, siendo un problema de salud pública a escala mundial, aproximadamente 134.490 casos nuevos se diagnostican cada año en EEUU. ${ }^{1}$ Representa uno de los principales tipos de cáncer en nuestro país, en varones se encuentra en tercer lugar después del cáncer de próstata y estómago; en mujeres ocupa el quinto puesto después del cáncer de mama, cuello uterino, tiroides y estómago.

El CCR es altamente heterogéneo en su fenotipo y genotipo, lo cual está relacionado con las diferentes vías de carcinogénesis descritas, que implican diferentes mecanismos de progresión y agresividad de la enfermedad.

Debido al potencial de cura en etapas tempranas de esta patología es importante conocer los grupos de alto riesgo, para realizar un diagnóstico oportuno y mejorar la sobrevida de estos pacientes.

\section{Etiología y epidemiología}

La literatura describe varios factores que incrementan el riesgo de CCR y que tienen influencia en el screening estos son: formas hereditarias de CCR, edad, grandes pólipos adenomatosos de alto riesgo, enfermedad inflamatoria intestinal y antecedente historia de irradiación.

El riesgo de desarrollar CCR incrementa con la edad, más del $90 \%$ de casos nuevos se manifiestan en pacientes mayores de 50 años. 
Entre los factores modificables se incluyen: obesidad, diabetes, alcohol, sedentarismo, consumo excesivo de carnes rojas y procesadas.

Además se describen factores protectores como los AINES-aspirina, los cuales son considerados como quimioprofilaxis en el síndrome de Lynch; medidas dietéticas como abolir el consumo de carnes procesadas y aumentar la ingesta de alimentos ricos en fibras vegetales y granos secos, ya que disminuiría el tiempo de contacto de las heces con la mucosa colónica.

\section{Bases Genéticas y biológicas}

EL CCR esporádico (en ausencia de historia familiar) supone el $90 \%$ de los casos, los síndromes de poliposis adenomatosa y el carcinoma hereditario no polipósico representan el 5 a $10 \%$.

Un sistema factores de pesos y contrapesos mantienen la homeostasis de la mucosa colorrectal, división celular y replicación; alteraciones en este sistema homeostático da lugar a cambios malignos, y están dados por múltiples procesos, en etapas que implican acumulación de cambios genéticos en el tiempo.

La pérdida de los genes importantes para el desarrollo de CCR se da por varios mecanismos, ya sea por una pérdida física de un segmento cromosomal debido a inestabilidad cromosómica, presente en $85 \%$ de pacientes con CCR, este es el mecanismo más común en los casos esporádicos; así mismo, se puede presentar otro mecanismo de daño genético que es el desajuste de la reparación del ADN (MMR) por la acumulación de secuencias repetidas de uno a cuatro nucleótidos (microsatélites) que provocan la función y acumulación defectuosa de los genes, este es el principal mecanismo del cáncer colorrectal hereditario no polipósico (síndrome Lynch). Finalmente, la 
hipermetilación de secuencias del ADN afecta la expresión de los genes y la traducción de la proteína, sin cambiar la secuencia de ADN silenciando su transcripción.

Las principales mutaciones de genes que intervienen en las bases moleculares del CCR a saber son: oncogenes (sobreexpresión de un gen o vía que estimula la proliferación celular incontrolada) los principales son RAS y MYC, mutación de genes supresores (provoca eliminación de la inhibición del crecimiento celular provocando crecimiento descontrolado celular) el APC es el gen más representativo y alteraciones en genes cuidadores (alteran la capacidad de reparar e inducir apoptosis).

La secuencia adenoma-adenocarcinoma descrita por Fearon y Vogelstein en 1990, como un proceso de múltiples pasos, en que cada evento genético acumulado confiere una ventaja de crecimiento selectivo a cada célula epitelial del colón, se evidencia debido a la frecuente yuxtaposición de tejido adenomatoso en regiones circundantes del adenocarcinoma.

\section{Presentación clínica}

Existen tres escenarios clínicos posibles: pacientes asintomáticos, pacientes con signos y síntomas sospechosos de CCR, y pacientes con cuadros de emergencia.

La mayoría de pacientes no presentan síntomas en etapas tempranas o estos son bastante inespecíficos, los pacientes que son diagnosticados en esta etapa, usualmente son resultado de los programas de investigación. Por el contrario, el 70 al 90\% de los pacientes presentan síntomas en etapas avanzadas, debido a la invasión de estructuras adyacentes o del lumen. ${ }^{2}$

En el contexto del cáncer de colón los síntomas dependen de la localización y del tamaño del tumor. Los síntomas más frecuentemente referidos son: hematoquesia $(37 \%)^{\mathbb{I}}$, cuanto más distal es 
el tumor, más sangre rojo brillante observaremos. El dolor abdominal $(34 \%)^{I}$ se presenta independiente de la localización, en fases tempranas por lo general es sordo y mal localizado, mientras que en fases tardías cuando el tamaño del tumor provoca obstrucción intestinal parcial el dolor se caracteriza por ser de tipo cólico, asociado con la ingesta de alimentos. Anemia microcítica hipocrómica de origen desconocido (23\%), más frecuente en tumores del lado derecho. Cambios de hábitos evacuatorios (segundo en frecuencia) ${ }^{3}$ se manifiesta más frecuentemente en tumores del lado izquierdo, ya sea por su calibre más estrecho y porque las heces están formadas, se manifiesta como disminución del calibre de las evacuaciones y por lo general aumentan en frecuencia.

Los síntomas menos comunes incluyen distención abdominal, nausea y vómito que pueden resultar en un cuadro obstructivo y en pérdida de peso.

Por otro lado, los tumores de recto se presentan con dolor rectal (cuando comprometen los esfínteres), tenesmo, rectorragia $(25 \%)^{1}$ y disminución del calibre de las evacuaciones en el caso de los tumores distales.

La pérdida de peso en ausencia de otros síntomas es un signo de mal pronóstico, así como la astenia, anorexia y debilidad que sugieren enfermedad metastásica.

El tacto rectal para las lesiones de recto inferior ( $7 \mathrm{~cm}$ bajo el margen anal), debe exponer la localización, morfología, número de cuadrantes envueltos, grado de fijación y movilidad.

El cáncer normalmente se propaga por vía linfática, hematógena o siembra peritoneal y los síntomas más frecuentes son el hepáticos, pulmonares y peritoneales; otros menos frecuentes son cerebrales y óseos. Aproximadamente el 20 al 25\% de pacientes se presenta con enfermedad metastásica al momento del diagnóstico, con síntomas constitucionales como pérdida de peso, 
caquexia, debilidad y anorexia ${ }^{4}$. Un nodo de Virchow (ganglio supraclavicular izquierdo) o nodo de la hermana Mary Joseph (nódulo umbilical) es otro hallazgo poco frecuente que se ha asociado con la diseminación a distancia del cáncer de colon.

\section{Diagnostico}

Los exámenes de diagnóstico dependerán del escenario clínico que presente el paciente, así tenemos tres escenarios: paciente en sospecha de CCR, diagnóstico descubierto en cribado o un paciente complicado.

La detección de sangre oculta se usa como método de cribado de CCR en mayores de 50 años, sin otros factores de riesgo la prueba de guyaco tiene la probabilidad de detectar el 5,1\% de CCR. La detección de sangre en heces con el test inmunohistoquímica usa anticuerpos monoclonales para hemoglobina humana es más específico para CCR y adenomas avanzados. Si es positiva se debería realizar una colonoscopía.

Alrededor del $30 \% 3$ de los pacientes con CCR son diagnosticados en ausencia de síntomas mediante endoscopía, que es el test más exacto y versátil para el diagnóstico, es el método de elección debido a su alta sensibilidad $(94,7 \%)$ en la detección de tumores y su capacidad de toma de biopsia, proporciona información precisa acerca de la mucosa de colón determinando las características de las lesiones. En colonoscopía tiene importancia para la detección de lesiones en el lado derecho y tumores sincrónicos. El $75 \%$ de lesiones cancerígenas aparecen en el colón descendente, sigma y recto, de ahí el valor diagnóstico de la rectosigmoideoscopía flexible que llega hasta el ángulo esplénico, sin embargo, hay que considerar que un $6 \%$ de lesiones cancerosas pasan desapercibidas por este método. 
El enema de bario es una técnica ampliamente disponible, pero carece sensibilidad y especificidad, puede detectar lesiones polipoideas (sobre todo en fase aérea) de $>1 \mathrm{~cm}$ en un 50 80\% de casos, el hallazgo característico en estadios más avanzados de CCR es la disminución del calibre de la luz concéntrica (signo de mordido de manzana).

La tomografía computarizada (TC), ampliamente utilizada por su disponibilidad y costo, permite una aproximación del estadio prequirúrgico en relación al tamaño, localización extensión local como metástasis a distancia sobretodo hepático. Permite la detección de adenomas y lesiones malignas de $>1 \mathrm{~cm}$ en el $67-94 \%$, y aumenta su especificidad de acuerdo al estadio y alcanzando un rango del $96-98 \%$.

La resonancia magnética tiene su valor diagnóstico al permitir delinear la extensión de la enfermedad, siendo además de elección para determinar la infiltración ganglionar sobretodo en el recto, pudiendo distinguir entre cambios fibróticos, tiene una exactitud de $85 \%$ para detectar metástasis hepáticas.

Los marcadores tumorales asociados con CCR son el antígeno carcinoembrionario (CAE) con una baja sensibilidad del $46 \%$, bajo rendimiento para seguimiento y pronóstico ${ }^{5}$.

Reportes de la clínica Lahey reportan en 10 años la distribución de los tumores siendo estos colon derecho $18 \%$, transverso $9 \%$, colon descendente $5 \%$ colon sigmoideo $25 \%$ y recto 43 $\%^{6}$.

\section{Estadio}

El sistema de clasificación de CCR preferido es el TNM (T profundidad del tumor $\mathrm{N}$ envolvimiento nodular y $\mathrm{M}$ de metástasis) desarrollado por Comité Americano de Tumores (AJCC) 
que en combinación determinan el estadio I al IV, cuya importancia es que determina el pronóstico y tratamiento.

Los factores que afectan el pronóstico en CCR son la histología pobremente diferenciada, diagnóstico en cuadro de emergencia como la perforación, invasión venosa, elevación del antígeno carcinoembrionario $(\mathrm{CEA})>5$ nanogramos / mililitro.

\section{Tratamiento}

La colonoscopia constituye el tratamiento estándar para pólipos malignos siempre y cuando sean localizados (que no penetre a la muscular mucosae). Las complicaciones de procedimientos de resección de lesiones polipoides son sangrado en $2 \%$ y perforación en $1 \%$ de los casos.

La piedra angular del tratamiento del CCR es la cirugía, pudiendo ser resuelto en totalidad cuando se diagnostica en etapas tempranas, cuya meta consiste en la resección del tumor primario en bloque con márgenes adecuados (mínimo de $2 \mathrm{~cm}$ microscópicamente sano) ${ }^{7}$ y resección de drenaje linfático (cólicos, paracolólicos e intermedios) de la zona comprometida, limitado mediante una resección segmentaria anatómica en función de su pedículo vascular a nivel de su raíz de origen.

El logro de estas metas es prevenir la recurrencia de esta enfermedad. Las resecciones extensas de colon junto con ganglios retroperitoneales no se recomiendan por que no añaden beneficio oncológico y aumentan sustancialmente las complicaciones operatorias. Así mismo, se debe explorar las vísceras en especial el hígado y superficie peritoneal con el objetivo de encontrar metástasis ya que se deberá determinar el grado de resecabilidad, si fuera posible en un solo tiempo o tomar la decisión de un procedimiento paliativo. 
Las opciones de resección dependen de la localización del tumor y del estadio en que se encuentre. En etapas más avanzadas se complementa con terapia adyuvante, mediante diferentes esquemas dependiendo el tipo de tumor, cada vez mejorando la sobrevida.

\section{Conceptos a tomar en cuenta para la cirugía:}

Es esencial un estadiaje prequirúrgico lo más exacto posible, para determinar la opción quirúrgica más adecuada.

Evaluación de un equipo multidisiplinario como coloproctólogo, oncólogo, patólogo y radiólogo.

Evaluación integral prequirúrgica tanto del estado general, nutricional y comorbilidades que puedan afectar la evolución del paciente.

El crecimiento tumoral intramural es el crecimiento tumoral tanto proximal como distal (longitudinal) a lo largo del colon que según reportes son no más que $2 \mathrm{~cm} 7$ a cada lado por que soporta la práctica de escisión de $5 \mathrm{~cm}$ o más de colon a cada lado disminuye el riesgo de recurrencia de anastomosis.

\section{Cáncer de Colon intraperitoneal y de Recto superior}

La resección o colectomía segmentaria y anastomosis primaria ya se con sutura mecánica o manual es el tratamiento de elección del cáncer colorectal y del recto superior.

Los tumores localizados en el ciego y colon ascendente debería ser resecado por hemicolectomía derecha, ligadura de ileocólica, cólica derecha y rama derecha de la cólica derecha es fundamental. Si le localiza el tumor en el ángulo esplénico se debería realizar una colectomía 
derecha extendida con ligadura de la cólica media cerca de su origen. Para lesiones de colon transverso una colectomía segmentaria de transverso con ligadura de la arteria cólica media. Para lesiones del ángulo esplénico se realiza una colectomía que involucra le mitad del transverso y anastomosis con la mitad del colon descendente siempre tomando en cuenta una movilización cuidadosa para evitar lesionar el bazo.

La hemicolectomía izquierda con resección de la mitad del colon transverso hasta el colon sigmoideo distal para tumores en el colon descendente. La ligadura alta de la arteria mesentérica inferior es fundamental. Para tumores localizado en el sigma-recto son removidos por una resección anterior (tumores por encima de la reflexión peritoneal).

\section{Recto medio e Inferior}

Los cánceres a $7-8 \mathrm{~cm}$ del margen anal tenemos algunas alternativas.

Resección Local: La resección Trasnanal tumores rectales a $10 \mathrm{~cm}$ del margen anal y en faces muy tempranas T1 son ideales esta vía de resección, cuyo objetico es la resección de la lesión con márgenes negativos en lesiones esta vía actualmente es discutido T2 ya que los resultados son aún poco satisfactores. Se extirpa la pared completa de la lesión con márgenes adecuados y sus indicaciones para lesiones menos de $4 \mathrm{~cm}$ con menos del $40 \%$ de la circunferencia dentro de los 6 cm distales. Así mismo están indicadas para cáncer más avanzadas en pacientes con comorbilidades serias. Se describen varias opciones como es la microcirugía endoscópica trasnanal (TEM), TAMIS

Resección radical: Para lesiones más avanzadas se puede realizar una resección anterior, una resección ultra baja con anastomosis a nivel coloanal siempre y cuando se maneje los principios oncológicos básicos. Todo esto es con fin de preservar la continencia dejando los esfínteres intactos 
a esto va enfocado el manejo moderno de los tumores de recto. Una de los gestos técnicos más importantes es la resección del mesorecto completo mismo que ha determinado un mejor pronóstico disminuyendo la tasa de recidivas.

La amputación abdominoperineal descrita por Miles. Es la extirpación del recto y ano por medio de una disección abdominal y perineal sacrificando los esfínteres así como la creación de una colostomía definitiva. Indicada para tumores que comprometen el esfínter y el recto distal.

Para los pacientes que han sido sometidos a una resección potencialmente curativa, se cree que la recurrencia de la enfermedad que surgen de micrometástasis clínicamente ocultas que están presentes en el momento de la cirugía alrededor del $25 \%$ y $50 \%$ presentes en estadios II y III . El objetivo de la terapia postoperatoria (adyuvante) es erradicar estas micrometástasis, lo que aumenta la tasa de curación. Está indicada en la etapa III de CCR (ganglios positivos ) en adelante y II cuando se cree que es de alto riesgo como número insuficiente de ganglios en la muestra, pobremente diferenciados histológicamente invasión linfo o vascular. El régimen estándar actualmente es a base de 5 - fluoracilo ( 5 FU ) y leucovorin así mismo la combinación de 5- FU / leucovorin / oxiplatino ( FOLFOX ) se utiliza como alternativa.

En cáncer de colon es infrecuente la recidiva local, en lesiones de recto la quimioterapia con radioterapia provee un control adecuado localmente y mejora la sobrevida.

Se usa radioterapia usar como tratamiento antes de la cirugía (neoadyuvancia) sobretodo en tumores de recto grandes cerca del esfínter anal con la finalidad de preservar esfínteres.

En los tumores de recto localmente avanzados, es decir, aquellos que alcanzan la grasa peri rectal o que tienen adenopatías en el meso- recto, la quimioterapia asociada a radioterapia 
neoadyuvante con posterior cirugía resectiva (EMRT), ha demostrado mejores resultados con menor número de complicaciones asociadas a la radioterapia que si el tratamiento se aplica después de la resección. En algunos estudios se ha asociado también a un aumento de la supervivencia.

La sobrevida a 5 años de acuerdo en CCR de acuerdo SEER (Surveillance, Epidemiology, and End Results) 7 entre 1991 y 2000 en 119363 casos son:

Etapa I (T1-2 N0) - 93,2 por ciento

Etapa IIA (T3N0) - 82,5 por ciento

Fase IIB (T4N0) - 72 por ciento

Fase IIIA (T1-2 N1) - 83 por ciento

Etapa IIIB (T3-4 N1) - 64 por ciento

Estadio IIIC (enfermedad N2) - 44 por ciento 
Franklin E. Encalada-Calero; Nelson X. Ramirez-García; Marlon K. López-Chinga

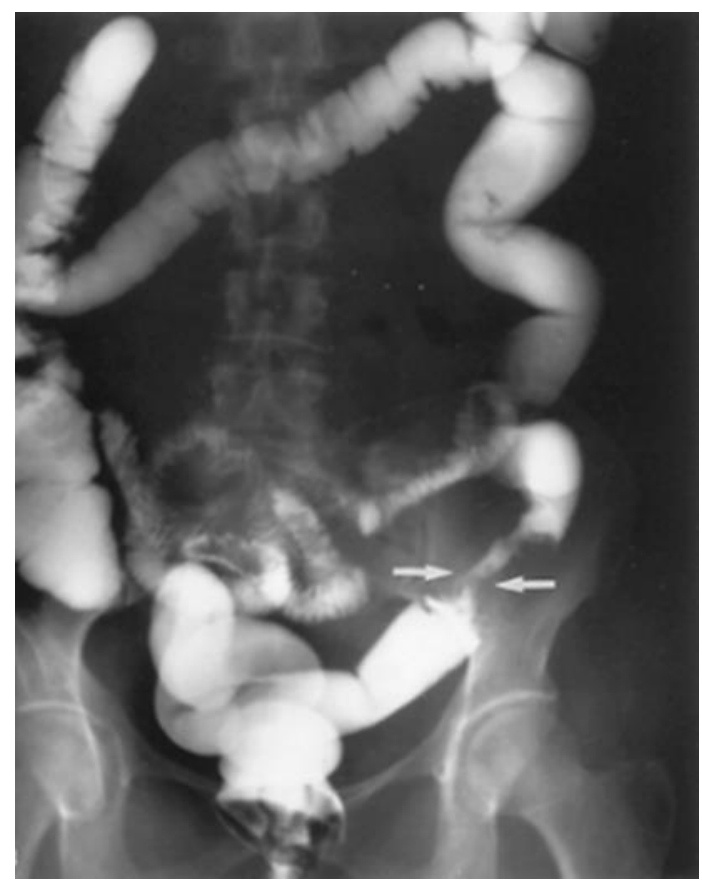

Figura $N^{\circ}$ 1.- Enema baritado de colon, en el que se observa área de estenosis por posible masa intracolónica.

Fuente: Nelson H, Petrelli N, Carlin A, et al. Guidelines 2000 for colon and rectal cancer surgery. J Natl Cancer Inst. 2001;93:583-96. 

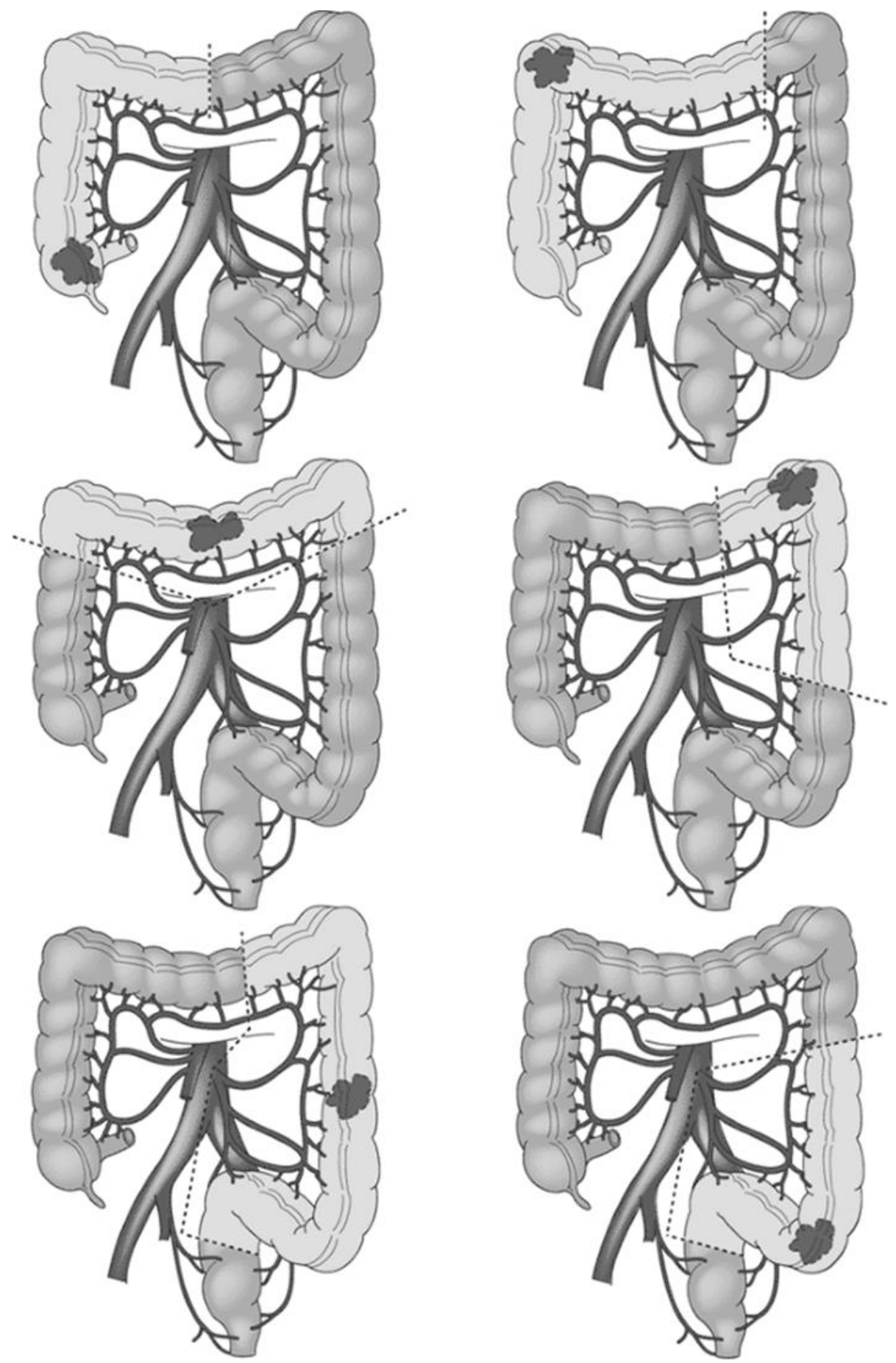

Figura $N^{\circ}$ 2.- Esquema que demuestra los diferentes lugares de resección colónica, dependiendo de la localización neoplásica.

Fuente: Nelson H, Petrelli N, Carlin A, et al. Guidelines 2000 for colon and rectal cancer surgery. J Natl Cancer Inst. 2001;93:583-96. 


\section{Bibliografía.}

1. Moreno CC, Mittal PK, Sullivan PS, et al. Colorectal Cancer Initial Diagnosis: Screening Colonoscopy, Diagnostic Colonoscopy, or Emergent Surgery, and Tumor Stage and Size at Initial Presentation. Clin Colorectal Cancer 2016; 15:67.

2. Appell MS. Fisiopatología, presentación clínica y el tratamiento de cáncer de colon. Gastroenterol Clin North Am. 2008; 37: 1-24. v.

3. David E. Beck, md, facs, fascrs Patricia L. Roberts, md, facs, fascrs Theodore J. Saclarides, md, facs, fascrs The ASCRS Textbook of Colon and Rectal Surgery Second Edition DOI 10.1007/9781-4419-1584-9

4. Cole SR, Young GP, Esterman A, Cadd A, Morcom J. A ran- domised trial of the impact of new faecal haemoglobin test technologies on population participation in screening for col- orectal cancer. J Med Screen. 2003;10:117-22.

5. Corman ML, Veidenheimer MC, Coller JA. Colorectal carci- noma: a decade of experience at the Lahey Clinic. Dis Colon Rectum. 1979;22:477-9.

6. Nelson H, Petrelli N, Carlin A, et al. Guidelines 2000 for colon and rectal cancer surgery. J Natl Cancer Inst. 2001; 93:583-96.

7. O'Connell JB, Maggard MA, Ko CY. Colon cancer survival rates with the new American Joint Committee on Cancer sixth edition staging. J Natl Cancer Inst 2004; 96:1420. 